\title{
Comparison of Different Kinds of Memory in Bipolar Disorder With or Without Obsessive- Compulsive Disorder Comorbidity
}

\author{
Alemeh Dehnabi', Elaheh Radfar², Hamid Radsepehr ${ }^{3 *}$ \\ ${ }^{1}$ MSc. of Nursing, Faculty of Jovein School of Nursing, Sabzevar University of Medical Sciences, Sabzevar, Iran \\ ${ }^{2}$ Sabzevar University of Medical Science, Sabzevar, Iran \\ ${ }^{2}$ Msc. of Clinical Psychology, Sabzevar University of Medical Science, Sabzevar, Iran
}

\begin{abstract}
Background: One challenging area in psychological pathology pertains to the comorbidity of the psychological disorders. Bipolar disorder can be pointed out among the most critical psychological diseases featuring common psychiatric comorbidity. The purpose of this study is to compare different kinds of memory in Bipolar Disorder with or without obsessive-compulsive disorder (OCD) comorbidity.

Methods: Sixty samples selected in 3 groups: 20 bipolar patients, 20 bipolar disorder with OCD comorbidity, and 20 healthy volunteers. Samples were selected using the available sampling method. Data collection tool included 3 questionnaires: Demographic Information Questionnaire, Comprehensive International Diagnostic Interview (CIDI), Wechsler's Memory Scale. Data analyzed by multivariate analysis of covariance and descriptive statistics.

Results: The results of multivariate analysis of variance analysis showed that at the level of 0.001 , there was a significant difference at least regarding one of the memory variables, between the subjects of the 3 groups of bipolar/OCD, bipolar disorder and healthy. According to the mean of the groups, the weakest function is related to patients with bipolar/OCD.

Conclusion: The results of the study showed that memory damage is more common in the bipolar/ OCD group, which should consider the treatment processes of these patients.

Keywords: Memory; Bipolar Disorder; Obsessive-Compulsive Disorder.
\end{abstract}

*Correspondence to Hamid Radsepehr, Msc. of Clinical Psychology, Sabzevar University of Medical Science, Sabzevar, Iran.

Tel: +989128508237

Email: h.radsepehr@gmail.com

Published online 20 March 2019

Citation: Dehnabi A, Radsepehr H. Comparison of different kinds of memory in bipolar disorder with or without obsessive-compulsive disorder comorbidity. Int Clin Neurosci J. 2019;6(1):6-10.

\section{Introduction}

According to Diagnostic and Statistical Manual of Mental Disorders, Fifth Edition (DSM-5), bipolar disorder is the condition in which the patient experiences one or several manic episodes, and sometimes also major depressive episodes. ${ }^{1}$ The prevalence of bipolar I disorder is estimated to be between $0 \%$ and $0.6 \% .^{2}$ This condition leads to a significant decrease in job performance, which in turn results in a lower socioeconomic status for the patients, despite having a similar level of education to that of their peers in the general population. ${ }^{2}$

Consequently, bipolar I disorder reduces the quality of life of the patients and imposes different costs on them, their families, and society. ${ }^{3,4}$ Mania is the primary clinical manifestation of bipolar I disorder. ${ }^{5}$ Patients in the manic phase diagnosed with bipolar I disorder, even when there are no depression episodes. ${ }^{2}$

Verbal memory distortion observed in a study conducted on individuals with bipolar disorder in acute and recovery states. ${ }^{6}$ Memory is a general concept referring to the group of the psychological events enabling an individual to store experiences and perceptions and recall them. ${ }^{7}$ The evidence from the studies carried out on the bipolar individuals is reflective of cognitive disorders in such areas as attention, working memory and performance. ${ }^{8}$ Cognitive disorders in bipolar patients caused by the disease's nature and the side effects of the drugs. ${ }^{9}$ Studies have demonstrated that the individuals who have bipolar disorder have cognitive disorders in both hypomanic and depression phases and these defections have continued at euthymic temperaments development. ${ }^{10}$ It concluded in a corticography of the bipolar disorder within the format of a neuropsychological meta-analysis that the disorder diagnosed in an equilibrium state with an intermediate level of neuropsychological damage to verbal memory and learning. ${ }^{11}$

One challenging area in psychological pathology pertains to the comorbidity of the psychological disorders. During recent years, high comorbidity of the psychological disorders, especially depression and anxiety disorders, has led to a search for mechanisms responsible for such comorbidity, including meta-diagnosis factors. ${ }^{12}$

(C) 2019 The Author(s). This is an open access article distributed under the terms of the Creative Commons Attribution License (http:// creativecommons.org/licenses/by/4.0/), which permits unrestricted use, distribution, and reproduction in any medium, provided the original work is properly cited. 
Bipolar disorder can be pointed out among the most critical psychological diseases featuring common psychiatric comorbidity. In line with this, it estimated that two third of the patients with bipolar disorder are also inflicted with a disorder of (DSM-I) I axis, as well. ${ }^{13}$ Moreover, findings are indicative of the idea that $20 \%$ of patients with bipolar disorder have lifelong comorbidities like obsessive-compulsive disorder (OCD). ${ }^{14}$

Alongside with these points, researches point to the interests stemming from meta-diagnosis processes for the improvement of perception and treatment of comorbidities and designing of therapeutic interventions. ${ }^{15}$ According to the materials mentioned above, the use of metadiagnosis mechanisms in psychological disorders can assist the therapists in the creation of effective treatment strategies for psychological disorders. Thus, considering the importance of the materials described above, the present study comparatively investigates memory in bipolar disorder with or without OCD comorbidity as well as in their healthy counterparts.

\section{Methods}

The present study is a psychological comparison of bipolar disorder with or without OCD comorbidity in memory function. This study is a comparative causal study. The statistical population included all individuals referring to Sabzevar Medical Sciences Centers in 2017. Samples included 20 bipolar patients, 20 bipolar disorder with OCD comorbidity, and 20 healthy volunteers from among the staff of the educational centers. Samples were selected using the available sampling method. All 3 groups matched according to demographic variables such as age, level of education, marital status, judges and duration of the disorder.

Inclusion criteria for patients in the study include age range of 20 to 40 years, having a minimum level of secondary education, Also, participants have a history of medication less than two years and have not received electrical shock until the time of research. In addition, the lack of first-degree relatives with schizophrenia, and other disorder of (DSM-I) I axis Based on the findings of the Comprehensive International Diagnostic Interview (CIDI), as well as Lack of history of brain damage, aphasia, physical and motor injury, epilepsy, stroke, tumor and mental retardation were among the other criteria for entering the study.

Besides the above-cited cases, a comprehensive international diagnostic interview was utilized to diagnose the patients with the disorder through an investigation of the existence of DSM-IV-TR criteria by experienced psychologists and psychiatrists from therapeutic centers. Individuals found disqualified for bipolar disorder, or comorbid bipolar and OCD disorders excluded from the study sample volume. The inclusion criteria for the evidence group were as follow healthy individuals who did not have a history of psychological disorder, cerebral and physical damage based on self-report and clinical interviews and no individuals with mental diseases found in their close relatives.

Before conducting the research, in order to observe the ethics of research, the research was explained to the participants and received informed consent from them. Data analyzed by multivariate analysis of covariance and descriptive statistics.

Data Collection Tools in this Study Included 3 Questionnaires

A. Demographic Information Questionnaire: This was a researcher-made questionnaire. There were questions about gender, age, marital status, employment, education, family income, suicide history, the age of onset of illness, duration of the illness, the name of the drug and its dosage.

B. CIDI: The questionnaire was a structured, standardized and comprehensive diagnostic interview used to assess psychiatric disorders. The World Health Organization recommends it, and reliable diagnoses could make regarding psychological disorders based on the definitions and scales given in DSM-IV-TR and ICD-10. Highly structured framework of CIDI makes the effect of the observer's information and interpretation minimized to the maximum possible extent. This leads to an increase in the questionnaire's reliability. ${ }^{16}$ Several studies in the international level have demonstrated acceptable to the excellent reliability of CIDI on an international level by various researchers for different cultures and times. ${ }^{16,17}$ In Iran, the validity and reliability of the instrument have respectively investigated by Amini et $\mathrm{al}^{16}$ and Davari Ashtiyani et al. ${ }^{17}$

C. Wechsler's Memory scale: the test has consisted of seven subscales each of which possesses its specific questions. Each subscale attributed to the assessment of an indicator of memory and the total sum of the scores gives the memory score. The test's reliability has been reported equal to 0.67 based on Cronbach a coefficient. Eight factors identified in an analysis of the test. ${ }^{18}$ According to the objective of the present study, use 4edfg made of only such subscales as pairwise verbal association (for the evaluation of verbal memory), logical memory (for an evaluation of verbal and auditory memory), forward and backward digital span (for the evaluation of auditory attention and short-term memory ability) and visual memory (for the evaluation of visual memory).

\section{Results}

The demographic characteristics of individuals shown in Table 1. The mean and standard deviation of memory types in the 3 groups shown in Table 2 .

Comparison of the meanings of Table 2 shows that they have a higher mean in all memory components, 
Table 1. Demographic Characteristics of Subjects in Terms of Sex, Education, and Marital Status

\begin{tabular}{lccc}
\hline Variable & $\begin{array}{c}\text { Bipolar } \\
\text { Disorder }\end{array}$ & $\begin{array}{c}\text { Bipolar / Obsessive } \\
\text { Compulsive Disorder }\end{array}$ & Healthy \\
\hline Sex & 9 & 12 & 10 \\
Female & 11 & 8 & 10 \\
Male & & & \\
Education & 9 & 7 & 6 \\
Diploma & 5 & 6 & 7 \\
Associate degree & 1 & 4 & 5 \\
$\quad$ Bachelor & & & 13 \\
Marital status & 12 & 13 & 7 \\
$\quad$ Single & 8 & 7 & \\
Married & & &
\end{tabular}

respectively, the healthy group, bipolar disorder, bipolar/ OCD. There is a difference between all the groups in memory performance averages.

As shown in Table 3, the significant levels of all tests indicate that there is a significant difference, at least regarding one of the dependent variables (one of the memory variables), between the subjects of the 3 groups of bipolar/OCD, bipolar disorder and healthy. According to Table 3, there is a significant difference between verbal memory and visual memory in the healthy group with bipolar/OCD and bipolar disorder. Also, there is a significant difference between the healthy group and bipolar/OCD in the logical memory variable. Also, the results indicate that there is a significant difference between verbal memory and logical memory between patients with bipolar/OCD and bipolar disorder. Concerning the counting of the reciprocal digits, the performance of the two groups of patients is weaker than the healthy group. However, there is only a significant difference between the bipolar/OCD group and the healthy group. No significant difference found between the studied groups in the field of counting forward figures.
Therefore, the research hypothesis confirms that there is a significant difference between the groups in memory performance. According to the mean of the groups, the weakest function is related to patients with bipolar/ OCD as these individuals show a weaker performance in verbal memory and logical memory and visual memory. Therefore, we conclude that memory damage is more common in the bipolar/OCD group.

\section{Discussion and Conclusion}

The present study findings indicated that there is a significant difference between individuals with bipolar disorder and their healthy counterparts regarding memory performance. Ebrahimi et al. Showed a significant difference in memory performance in their study on the comparison of memory types in different groups (OCD, schizophrenia, bipolar disorder, and normal people). As individuals with schizophrenia have weaker performance in verbal memory, bipolar individuals in logical memory and OCD in visual memory. This is consistent with the results of the present study regarding the memory performance of disorders inpatient group as compared to the healthy group.

In line with the prior research, the present study, as well, figured out verbal memory destructions in patients with bipolar disorder. The findings of the present study demonstrated that the performance of the patients with bipolar disorder has been significant in the backward repetition of the numbers and this is in compliance with what has found before. As for the visual memory performance, the present study findings indicated that there is a significant difference between the bipolar patients' visual memory scores and those obtained for the healthy individuals' group and this same finding can also see in the previous studies. ${ }^{6,19,20}$ Moreover, some studies ${ }^{21-24}$ indicated that individuals with bipolar disorder, as compared to healthy individuals, have more performance dysfunction. Zarekar et al, as well, showed in

Table 2. Mean and Standard Deviation of Wechsler Memory

\begin{tabular}{|c|c|c|c|c|}
\hline Variable & Group & Average & Standard Deviation & Number \\
\hline \multirow{3}{*}{ Verbal memory } & Bipolar/obsessive compulsive disorder & 14.65 & 3.37 & 20 \\
\hline & Bipolar disorder & 15.55 & 2.13 & 20 \\
\hline & Healthy & 20.15 & 2.03 & 20 \\
\hline \multirow{3}{*}{ Logical memory } & Bipolar/obsessive compulsive disorder & 13.70 & 2.40 & 20 \\
\hline & Bipolar disorder & 14.80 & 2.54 & 20 \\
\hline & Healthy & 20.25 & 1.97 & 20 \\
\hline \multirow{3}{*}{ Counting forward figures } & Bipolar/obsessive compulsive disorder & 6.35 & 4.13 & 20 \\
\hline & Bipolar disorder & 6.60 & 3.47 & 20 \\
\hline & Healthy & 7.75 & 2.46 & 20 \\
\hline \multirow{3}{*}{ Counting numbers to back } & Bipolar/obsessive compulsive disorder & 4.35 & 1.66 & 20 \\
\hline & Bipolar disorder & 4.95 & 1.50 & 20 \\
\hline & Healthy & 5.75 & 1.65 & 20 \\
\hline \multirow{3}{*}{ Visual memory } & Bipolar/obsessive compulsive disorder & 7.05 & 1.31 & 20 \\
\hline & Bipolar disorder & 8.65 & 1.84 & 20 \\
\hline & Healthy & 14.00 & 2.02 & 20 \\
\hline
\end{tabular}


Table 3. Multivariate Analysis of Variance (MANOVA) Test for Memory Scores

\begin{tabular}{llllll}
\hline Exam Name & Amount & Hypothesis Df & Error Df & F & P \\
\hline Pillai's Trace & 1.032 & 15.000 & 222.000 & 7.756 & 0.000 \\
wilk's Lambda & 0.152 & 15.000 & 199.162 & 13.023 & 0.000 \\
Hotelling's Trace & 4.399 & 15.000 & 212.000 & 20.726 & 0.000 \\
Roy's Largest root & 4.110 & 5.000 & 74.000 & 60.831 & 0.000 \\
\hline
\end{tabular}

a comparison of executive function, emotional intelligence and emotion regulation of individuals with schizophrenia, bipolar disorder and healthy counterparts that the first two significantly differ from the healthy individuals in all indicators of executive function. ${ }^{25}$ It could state in an elaboration of this finding that the patients with bipolar disorder also have essential defections in their prefrontal lobe. ${ }^{26}$ It assumed that the cognitive processes highly associated with the precise functioning of the prefrontal cortex. Damage to prefrontal lobe diminishes the ability to make the plan and stay organized. Thus, the bipolar patients feature lower levels of executive functions concerning healthy individuals.

Bigdeli et al, as well, in a study under the title of "comparing the cognitive flexibility, mind theory and working memory in university students with OCD and healthy group" showed that the individuals with OCD are weaker than the healthy students in cognitive flexibility and working memory. ${ }^{27}$ This is following what has found herein. Mahmood Alilou et al, as well, indicated in a study of reliance on memory and memory errors in individuals with OCD in comparison to those in healthy individuals that the OCD group testees, in contrast to normal testees, enjoy lower memory reliance. Furthermore, the memory error was found higher in the OCD group testees than in normal testees. The results were suggestive of the idea that there are a lower memory reliance and higher memory error in OCD testees. ${ }^{28}$ Therefore, OCD plays a considerable role in the weakening of cognitive processes and memory function and this is generally similar to what has found in the present study regarding the memory performance reduction in patient group in respect to the healthy group.

Hassani et al, as well, in a study on the cognitive emotion regulation, anxiety and impulsiveness strategies in bipolar disorder with and without OCD showed that the bipolar patients with OCD make more frequent use of maladaptive strategies like self-reproach and otherreproach in comparison to healthy individuals. Both of the patient groups were found making greater use of rumination, and catastrophizing strategies in respect to the healthy individuals and the bipolar patients with OCD obtained higher scores in these strategies in contrast to only bipolar patients. Furthermore, higher scores also evidenced for both of these groups in respect to healthy individuals in such variables as anxiety as well as lack of cognitive-attention, motor, and impulsive planning whereas the mean scores of the patients with comorbidity of OCD and bipolar disorder were higher than those of only bipolar patients. ${ }^{29}$ Thus, it seems that the individuals with OCD and bipolar disorder comorbidity are generally more deficient in cognitive functions and emotion regulation and this conforms to the present study results indicating the intergroup differences regarding general functioning of memory in such a way that a higher level of disorder was also evidenced herein in comorbidity group.

Overall, according to the findings of the present study, memory damage is more common in the bipolar/ OCD group than other groups. Limitations of research include failure to measure patient intelligence, lack of precise control of the dose of medications used; Failure to investigate the possible effects of drugs in cognitive processes, low volume, and availability of sampling. For this purpose, it is suggested to use in future research in other diseases with bipolar disorder, Determine the severity of the disease and the dominant phase in bipolar disorder in patients, Determination of patients' iQ, selection of more sample size and random sampling.

\section{Conflict of Interest Disclosures}

The authors declare that they have no conflict of interests.

\section{Ethical Statement}

The researcher explained the objectives of the study to the participants and received informed consent from them. Also emphasized the confidentiality of patient information.

\section{Acknowledgments}

In this regard, the authors of the article are grateful to all of the managers and staff working in the educational and medical centers of Sabzevar Medical Sciences who helped them in carrying out the research. It is also grateful to all respected patients who gave their time to the researchers.

\section{References}

1. Whitbourne SK, Halgin R. Abnormal psychology: Clinical perspectives on psychological disorders with DSM-5 update. New York: McGraw-Hill Education; 2014.

2. American Psychiatric Association. Diagnostic and statistical manual of mental disorders (DSM-5®). Missouri: American Psychiatric Pub; 2013.

3. van der Voort TY, van Meijel B, Hoogendoorn AW, Goossens PJ, Beekman AT, Kupka RW. Collaborative care for patients with bipolar disorder: Effects on functioning and quality of life. J Affect Disord. 2015;179:14-22. doi: 10.1016/j. jad.2015.03.005.

4. Sammarco A. Women's health issues across the life cycle. Burlington: Jones \& Bartlett Publishers; 2016.

5. Proudfoot J, Doran J, Manicavasagar V, Parker G. The precipitants of manic/hypomanic episodes in the context of bipolar disorder: a review. J Affect Disord. 2011;133(3):3817. doi: 10.1016/j.jad.2010.10.051.

6. Gharaeipour M. Neuropsychological function and its relationship with psychosocial function and clinical features in Bipolar Disorders. Psychosocial Studies. 2007;3(1):73-84. [Persian].

7. Pourafkary N. A comprehensive dictionary of psychology \& psychiatry. Tehran: Farhangmoaser; 2007.

8. Sachs G, Schaffer M, Winklbaur B. [Cognitive deficits in 
bipolar disorder]. Neuropsychiatr. 2007;21(2):93-101.

9. Wingo AP, Harvey PD, Baldessarini RJ. Neurocognitive impairment in bipolar disorder patients: functional implications. Bipolar Disord. 2009;11(2):113-25. doi: 10.1111/j.1399-5618.2009.00665.x

10. Malhi GS, Ivanovski B, Hadzi-Pavlovic D, Mitchell PB, Vieta E, Sachdev P. Neuropsychological deficits and functional impairment in bipolar depression, hypomania and euthymia. Bipolar Disord. 2007;9(1-2):114-25. doi: 10.1111/j.13995618.2007.00324.x

11. Kurtz MM, Gerraty RT. A meta-analytic investigation of neurocognitive deficits in bipolar illness: profile and effects of clinical state. Neuropsychology. 2009;23(5):551-62. doi: 10.1037/a0016277.

12. McLaughlin KA, Nolen-Hoeksema S. Rumination as a transdiagnostic factor in depression and anxiety. Behav Res Ther. 2011;49(3):186-93. doi: 10.1016/j.brat.2010.12.006.

13. Bellani M, Hatch JP, Nicoletti MA, Ertola AE, Zunta-Soares G, Swann AC, et al. Does anxiety increase impulsivity in patients with bipolar disorder or major depressive disorder? J Psychiatr Res. 2012;46(5):616-21. doi: 10.1016/j. jpsychires.2012.01.016.

14. Amerio A, Odone A, Marchesi C, Ghaemi SN. Treatment of comorbid bipolar disorder and obsessive-compulsive disorder: a systematic review. J Affect Disord. 2014;166:25863. doi: 10.1016/j.jad.2014.05.026.

15. Gruber J, Eidelman P, Harvey AG. Transdiagnostic emotion regulation processes in bipolar disorder and insomnia. Behav Res Ther. 2008;46(9):1096-100. doi: 10.1016/j. brat.2008.05.004.

16. Amini H, Alaghbandrad J, Sharifi V, Davari-Ashtiani R, Kawyani H, Shahrivar Z, et al. Validity of a Farsi translation of the composite International Diagnostic Interview (CIDI) to diagnose schizophrenia and bipolar disorder. Tehran University Medical Journal. 2006;64(8):31-42. [Persian].

17. Davari Ashtiyani R, Alaghbandrad J, Sharifi V, Amini H, Kawyani H, Shabani A, et al. Reliability of the Persian version of the Comprehensive International Diagnostic Interview (CIDI) to diagnose schizophrenia and bipolar disorder. Advances in Cognitive Science. 2004;6:1-9. [Persian].

18. Orangi M, Atefvahid MK, Ashayeri H. Standardization of the revised Wechsler memory scale in Shiraz. Iranian Journal of Psychiatry and Clinical Psychology. 2002;7(4):56-66. [Persian].

19. Ebrahimi E, Roostaei M, Ghadiri M, Rayat Moeni F. Comparison of memory in practical obsessive-compulsive disorder, schizophrenia, bipolar, and normal people. The first scientific conference on psychology, education and pathology of society, electronically, Green Gold Company, Payesh Forum; 2015. https://www.civilica.com/PaperASIBCONF01 ASIBCONF01_031.html.

20. Karimi Aliabad T, Kafi M, Farrahi H. Study of executive function in bipolar disorder patients. Advances in Cognitive Science. 2010;12(2):29-39. [Persian].

21. Kurtz MM, Gerraty RT. A meta-analytic investigation of neurocognitive deficits in bipolar illness: profile and effects of clinical state. Neuropsychology. 2009;23(5):551-62. doi: 10.1037/a0016277

22. Krzystanek M, Krzeszowski D, Jagoda K, Krysta K. Long term telemedicine study of compliance in paranoid schizophrenia. Psychiatr Danub. 2015;27 Suppl 1:S266-8.

23. Hill SK, Reilly JL, Keefe RS, Gold JM, Bishop JR, Gershon ES, et al. Neuropsychological impairments in schizophrenia and psychotic bipolar disorder: findings from the BipolarSchizophrenia Network on Intermediate Phenotypes (B-SNIP) study. Am J Psychiatry. 2013;170(11):1275-84. doi: 10.1176/ appi.ajp.2013.12101298.

24. Hasenkamp W, James GA, Boshoven W, Duncan E. Altered engagement of attention and default networks during target detection in schizophrenia. Schizophr Res. 2011;125(23):169-73. doi: 10.1016/j.schres.2010.08.041.

25. Zarekar A, Rahimiyambogar E, Ghodrati M. Comparison of Executive Function, Emotional Intelligence and Emotion Regulation in Schizophrenia and Bipolar Disorders and Healthy People. Journal of Ilam University of Medical Sciences. 2015;23(4):123-34. [Persian].

26. Clark L, Iversen SD, Goodwin GM. A neuropsychological investigation of prefrontal cortex involvement in acute mania. Am J Psychiatry. 2001;158(10):1605-11. doi: 10.1176/appi. ajp.158.10.1605.

27. Bigdeli I, badin M, Sabahi P. The comparison of cognitive flexibility, theory of mind and working memory in students with symptoms of obsessive-compulsive disorder and normal group. Advances in Cognitive Science. 2017;18(4):24-37. [Persian].

28. Mahmood Alilu M, Hashemi T, Esmaeel Zade H. Confidence in memory and memory impairment in people with obsessivecompulsive states compared to normal people. Journal of Psychological Studies. 2014;9(4):60-71. [Persian].

29. Hassani J, Ariana Kia E. Cognitive Emotion Regulation Strategies, Anxiety and Impulsivity in Bipolar Disorder with and without Comorbid Obsessive-Compulsive Disorder. Iranian Journal of Psychiatry and Clinical Psychology. 2016;22(1):39-49. [Persian]. 